\title{
Pedestrian vision and collision avoidance behaviour: Investigation of the Information Process Space of pedestrians using an eye tracker
}

\author{
Kay Kitazawa ${ }^{1}$, Taku Fujiyama ${ }^{2}$ \\ ${ }^{1}$ Centre for Advanced Spatial Analysis (CASA), University College London \\ 1-19 Torrington Place, London, UK WC1E 7HB \\ email: k.kitazawa@ucl.ac.uk \\ ${ }^{2}$ Centre for Transport Studies, University College London \\ Gower Street, London WC1E 6BT \\ email: taku@transport.ucl.ac.uk
}

Summary. This study investigates the Information Process Space (IPS) of pedestrians, which has been widely used in microscopic pedestrian movement simulation models. IPS is a conceptual framework to define the spatial extent within which all objects are considered as potential obstacles for each pedestrian when computing where to move next. The particular focus of our study was identifying the size and shape of IPS by examining observed gaze patterns of pedestrians. A series of experiments was conducted in a controlled laboratory environment, in which up to 4 participants walked on a platform at their natural speed. Their gaze patterns were recorded by a head-mounted eye tracker and walking paths by laser-range-scanner-based tracking systems at the frequency of $25 \mathrm{~Hz}$. Our findings are threefold: pedestrians pay much more attention to ground surfaces to detect immediate potential environmental hazards than fixating on obstacles; most of their fixations fall within a cone-shape area rather than a semicircle; and the attention paid to approaching pedestrians is not as high as that paid to static obstacles. These results led to an insight that the structure of IPS should be re-examined by researching directional characteristics of pedestrians' vision.

Key words. Pedestrian vision, collision avoidance, Information Process Space

\section{Background}

Following the trend of sustainable development, pedestrian-oriented planning has started attracting much attention in several discourses; transport studies, urban planning and architecture. A recent trend in this subject is to develop predictive models of pedestrian movement. The subject of how people move around encompasses a huge variety of activities, ranging from migration and commuting movement between cities to how they manoeuvre themselves in crowds. This paper focuses on pedestrian movement at the smallest scale, where the individual pedestrians' movement patterns, more specifically how they avoid bumping into each other or how they avoid obstacles, are analyzed. At this "microscopic" level there has been a recent surge of studies that utilize disaggregated models that represent pedestrians' dynamics as a series of interactions between individual pedestrians' behaviour [1-10]. 
Although these models differ in terms of their spatial representation (i.e. discrete space or continuous space) as well as in terms of the techniques used to incorporate the behaviour model into computational codes, they share a common element in modelling individual pedestrians' behaviour: Information Process Space. IPS is essentially an approximation of the computational scope limitation in the environment for which modellers assume interactions between any given pedestrian and other pedestrians or obstacles. In a collision avoidance scenario, it corresponds to how far each individual pedestrian pays attention when he/she decides where to move next to avoid another pedestrian.

IPS is conceptualized in various different ways. Most Cellular Automata (CA) based pedestrian behaviour models represent pedestrians' walking paths as a chronological transition of the state of cells in a regular 2D space lattice. In CA, the cell states are simultaneously updated in discrete time steps and the change of the state of each cell depends on some local rules defined solely based on its own previous state and that of its neighbouring cells. At every step, each of the neighbouring cells is examined as a potential next destination in terms of whether it is occupied by other pedestrians or obstacles and thus whether potential collision may occur. Therefore 'neighbourhood' is used synonymously in this context for ISP, and is modelled as an extension of Moore neighbourhood in which the number of cells $n$ in the neighbourhood of cell $x(x$ included $)=(2 r+1)^{2}$ as shown in Figure 1. Pedestrians' natural walking speed is taken into account for the selection of radius $r[8,11]$. This type of ISP allows pedestrians to "perceive" all the information from different directions equally.

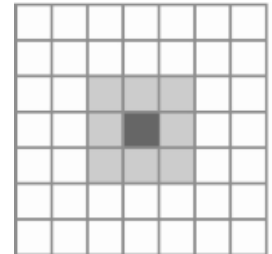

(a)

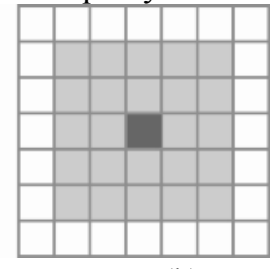

(b)

Fig. 1 IPS represented in Cellular Automata-based models; (a) $r=1$; (b) $r=2$

Other models use the analogy of the human field of vision to define the topography of neighbourhood. A viewshed or Isovist is an area of continuous space that is visible from a fixed vantage point as shown in Figure 2 (a). Visual neighbourhood used by Turner and Penn [12] interprets viewsheds into 2D discrete space and defines a neighbourhood relationship for any given location on the grid-shape graph as shown in Figure 2(b). ISP here is a collection of directly visible vertices each of which receives pedestrians' attention equally regardless of the walking direction in the same way as the neighbourhood cells do in CA-based models.

(a)

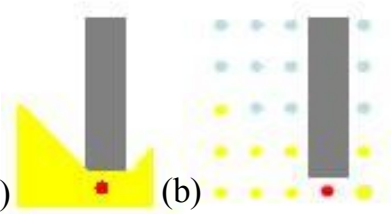

Fig. 2 IPS represented by viewshed (a) and visual neighbourhood (b) 
The aforementioned two models assume an even distribution of pedestrians' attention to the surrounding environment. There are several models that allow its uneven distribution. Utility-maximization-based models regard pedestrian behaviour as an optimization process of the cost functions for each alternative walking path. The model proposed by Hoogendoorn et al $[5,13]$ applies a three-tiered structure for the utility calculation: strategic, tactical and operational levels. The first strategic level concerns the choice of general behavioural and activity area. It sets an activity for a pedestrian to perform, for which he or she needs to make decisions optimizing expected subjective utility. The second level is for way-finding to reach the activity area chosen in the previous process. It is in the third operational level, where a pedestrian computes desired velocity at each time instant and location based on kinematic costs and psychological discomfort, that ISP is implemented. The negative utility (cost) due to walking too close to obstacles and other pedestrians is integrated into the calculation by applying a scaling parameter that describes the proximity discomfort reduction rate as a function of the distance between two objects; in other words, pedestrians and obstacles in the vicinity yield stronger negative proximity cost than those in the distance. The parameters are called region of influence of obstacle and spatial discount factor, respectively. These parameter values are further influenced by two anisotropy factors [5]. They are the abstract form of the biased pedestrians' perception of the objects which are located in front of and behind them as shown in Figure 3, and are provided as the ratio to the perception level of the stimuli coming from their sides, which is given as 1 . The "behind" anisotropy can be interpreted as how much sensory information other than optical data (e.g. sounds) pedestrians use for negotiating their way in collision avoidance behaviour.

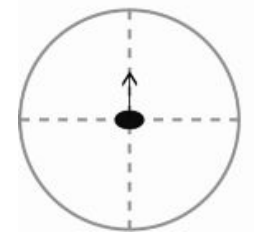

(a)

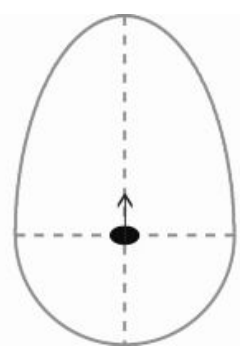

(b)

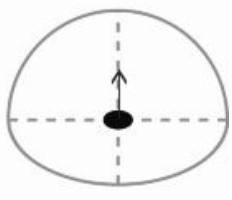

(c)

Fig. 3 Even and uneven distribution of pedestrians' perception represented with three different values for the front and back anisotropy factors; (a) $\mathrm{ff}=$ $\mathrm{fb}=1$; (b) $\mathrm{ff}=2, \mathrm{fb}=1$; (c) $\mathrm{ff}=1, \mathrm{fb}=0.5$.

Similar parameters are adopted by Social Force models $[9,10]$ to accommodate the difference in pedestrians' perception of other pedestrians in relation to those approaching from the front or those approaching from behind. Social Force-based models that represent crowd motion by interaction of particles according to the laws of Newtonian mechanics were first proposed by Helbing et al [14]. The fundamental concept of this type of model is that pedestrians feel and exert social force, either repulsive or attractive, on each other. The size and the shape of ISP is represented in the form of a parameter called Fall-Off Length of Social Repulsion Force weighted by two coefficients that determine which of the face-to-face and 
face-to-back forces will be used in the computation of the strength of social force based on the relative angle of their body directions.

The analogy of human vision field for IPS is more explicitly used in other models $[2,4]$ by setting a fan-shaped area around the body of an individual pedestrian. The area is a direct approximation of the field of vision of pedestrians (FOV), which is defined by its sectional angle and radius as shown in Figure 4. The difference between FOV and the aforementioned visual neighbourhood lies in the definitive shape of FOV, which is based on the cognitive studies on the physical characteristics of human perceptions. Unlike other models, pedestrians' attention ranges only to the front and partially to both sides of the body in the FOV-based models.

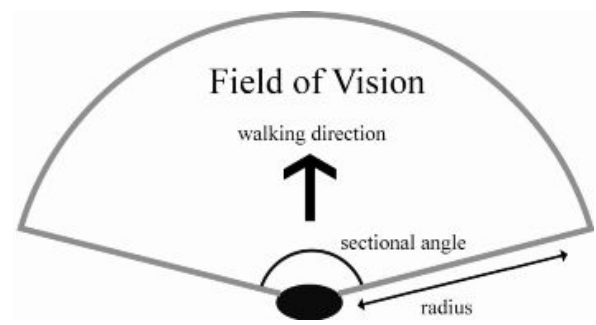

Fig. 4 Field of vision around a pedestrian

Despite the fact that IPS forms the core of most microscopic pedestrian behaviour models, neither its validity nor the details of the parameters of IPS have been fully examined by comparison with actual data. Consequently the focus of previous microscopic pedestrian behaviour models was on recreating "realistic" movement patterns with a selected number of pre-defined behaviour rules. The assessment of the behaviour rules is based solely on its performance, which is subjectively evaluated from the viewpoint of visual similarity with reality. This is partly due to the difficulties in obtaining datasets of pedestrian movements at a scale fine enough to analyze the details of IPS.

This study utilizes an automatic tracking system based on laser range scanners to document pedestrians' walking trajectories as well as utilizing a head-mounted eye tracking device to register their gaze patterns while walking. The application of such technologies is expected to be useful for the investigation of ISP. Our particular interests are in measuring its size and shape as well as identifying any potential environmental factors that exercise influence on it. Our research questions include:

1. What is the distance between a pedestrian and an obstacle when he or she first pays attention to it?

2. How long does a pedestrian look at an obstacle while avoiding it?

3 . Are there any variations in the result of question 1 between when the participant avoids static obstacles and when he or she avoids other pedestrians?

4. Are there any variations in the result of question 2 between when the participant avoids the static obstacles and how long he or she looks at other pedestrians? 


\section{Methodologies}

The datasets used for this study are taken from two-day observation studies of pedestrian microscopic movement that were conducted at the Pedestrian Accessibility and Movement Environment Laboratory (PAMELA) at University College London. The surveys consisted of sets of walking sessions on the platform (WL: $3.6 \mathrm{~m} \times 15.6 \mathrm{~m}$ ) in PAMELA. There were two types of platform setting: with and without two mannequins used as obstacles (WLH: $0.4 \mathrm{~m} \times 0.4 \mathrm{~m} \times 1.5 \mathrm{~m}$ ) placed in the middle of the platform. he number of pedestrians on the platform also varied from 1 to 4 . In each session, the participants were instructed to walk at natural speed from one end of the platform to the other and to repeat until receiving a further instruction to stop. Each session took approximately two minutes. Their positions on the platform were observed manually and also recorded by infra-red laser range scanners (LD-A Maker manufactured by IBEO Automobile Sensor, Germany) every 0.1 second. The scanners were pre-installed on the outer wall of the platform as part of PAMELA facilities. The information was sent from the scanners to a computer via Ethernet cable. A piece of custom-made software provided by the company arsenal research $\mathrm{GmbH}$ (Austria) was used to extract each pedestrian's walking path on the platform from the timeseries laser point dataset.

Amongst the participants in each session, one person (three in total for the whole study) wore an eye tracker. The eye tracker used was an iViewX Head Mounted Eye Tracking device (HED) manufactured by SensoMotoric Instruments GmbH (Germany). The device was to measure the gaze position of the participant, which is a point in a person's field of view. It consists of a lightweight helmet, an eye movement tracking camera, and a scene camera that captures the field of view. The gaze position is provided every 0.04 second ( 25 frames per second) in the form of a cursor inside Bitmap images (see Figure 5).

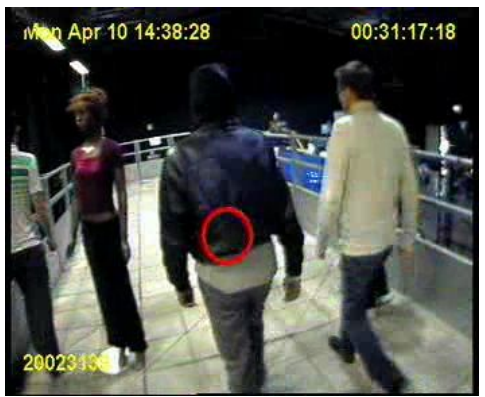

Fig. 5 An example of the field of view recorded by Eye tracker

The eye movement data and positional data (re-sampled and interpolated at the interval of 0.04 seconds) were then integrated by time-synchronization processes. A continuous gaze at the same point for more than 0.08 of a second was regarded as a fixation. If the gaze point in the next frame fell on the same object and within the range of 3.0 degrees (roughly $10 \mathrm{~cm}$ ) from the axis to the original gaze position, the participant was regarded as gazing at the same point (and thus fixating). The threshold values and other definitions of pedestrians' fixation in this study follow those in a previous study [15]. All the fixations were identified with time information (when it occurred), 
location (where on the platform it occurred), and the type of object (e.g. other pedestrians, mannequins, etc).

\section{Results}

\subsection{General fixation behaviour}

During the observation, the following points were found:

- Fixation on the static obstacles on the platform was often observed at the beginning of each walking session

- Fixation on the platform surface was often observed during the walking session

- Fixation on the parapet (hand rail of the platform) located around the perimeter of the platform at waist level was observed

These results vary from those of other pedestrian simulations due to the fact that few of them take account of objects other than fellow pedestrians or boundary walls.

\subsection{Fixations by the object type}

Figure 6 shows the observed fixations classified into 7 different categories: other pedestrians in the same walking session, side and facing parapets, platform surface, static objects (mannequins), and other objects such as a building wall, survey staff, and the area outside of the platform. "No gaze" includes gazes that did not last for two or more frames, which means that their duration was less than our definition of fixation. "No gaze" also includes observations in which the eye tracking system could not analyse where the participant gazed. Figure 6 clearly illustrates that the participants often fixated on the platform surface as well as on other objects on the platform.

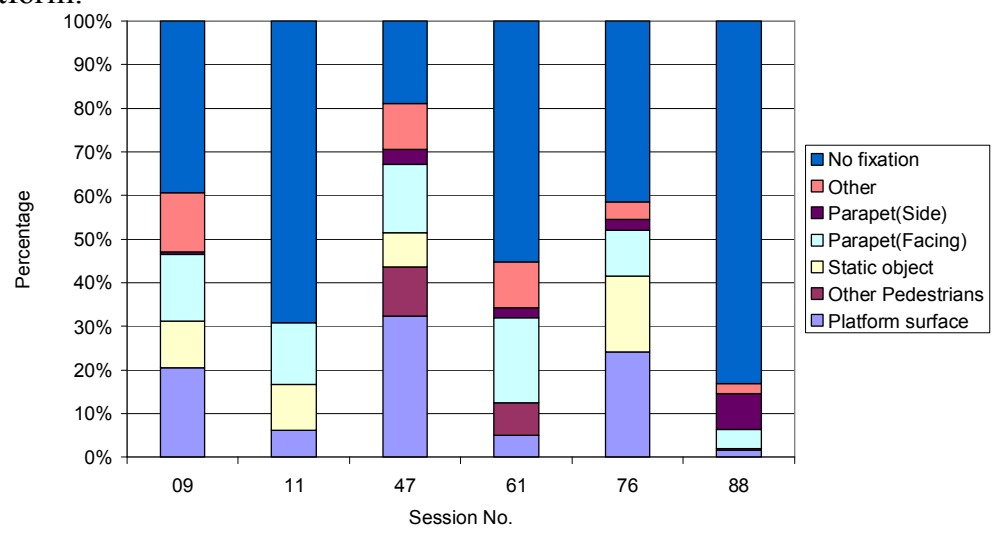

Fig. 6 Observed fixations by object types

\subsection{Location of the observed fixations}

Figure 7 shows the fixation at each point along the walking path of a pedestrian in one of the sessions (session 09). Point $(0,0)$ in the figure indicates the origin of our coordinate system on the platform. It is located at one end of the long side of the platform (x-axis) and in the middle of its short side (y-axis). The figure illustrates that the participant started fixating on a 
static obstacle (mannequin) at a distance of several meters when approaching it.

Figure 8 shows the numbers of observed fixations on a static obstacle in relation to distances between the participant and the obstacle. The data used for this analysis includes all the fixations in all the sessions of the experiment The fixations were aggregated and divided into the distance categories. For instance, category " 0 " includes the fixations with the distance value between 0.00 and $0.99(\mathrm{~m})$.

On the other hand, Figure 9 shows the numbers of observed fixations on another participant in relation to distances between the fixated participant and the eye-tracker participant. In this analysis, we did not distinguish between oncoming participants and leading participants, who walked in front of the eye-tracker participant and in the same direction. Comparison between the two figures leads us to notice that distances at which the participant fixated on another participant were shorter than those on a static obstacle.

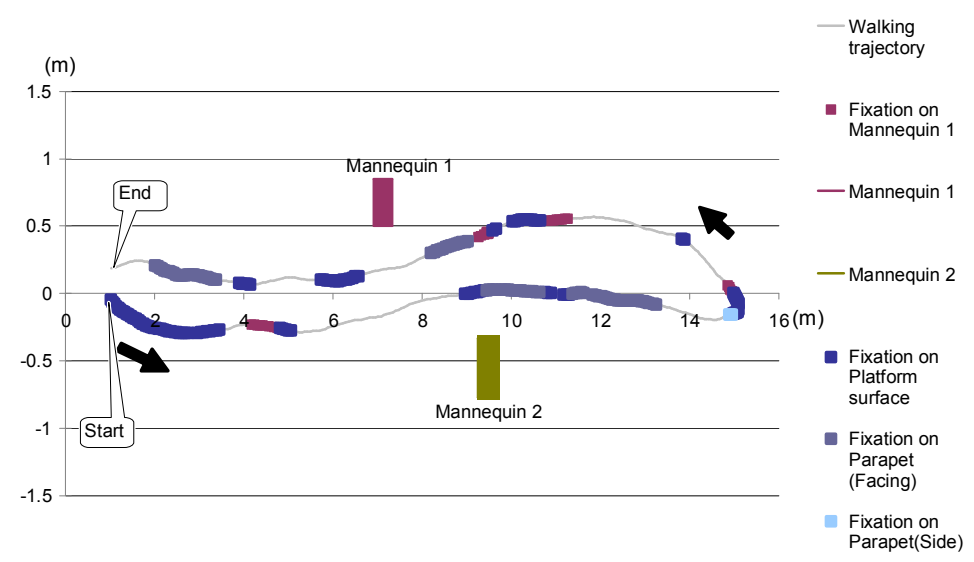

Fig. 7 Trajectory and fixation object in Session 09

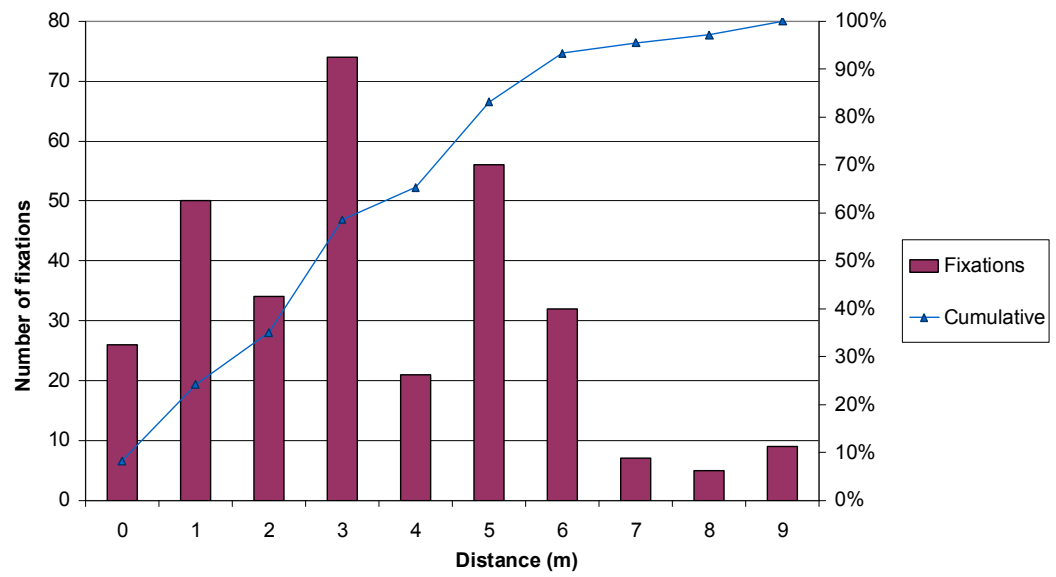

Fig. 8 The number of observed fixations on a static obstacle by distance 


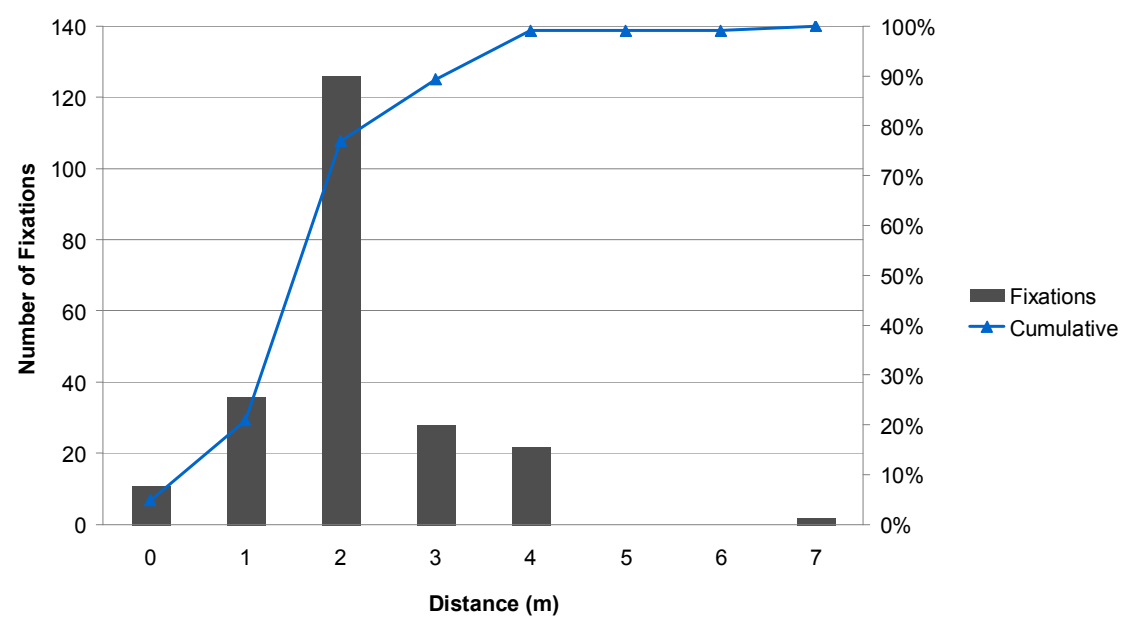

Fig. 9 The number of observed fixations on other pedestrians by distance

Table 1 shows the average distances at which the eye-tracker wearers first fixated on the other pedestrians who were walking in the same direction in front (leading participants), on those who were approaching head-on, and on the static obstacles. From all the fixations, the location and time information of the first frame was extracted to calculate the distance between the participant and the object. Table 1 indicates that the participant fixated on a static obstacle at the greatest distance, followed by an approaching participant and then a leading participant.

Table 1 The number of observed fixations on other pedestrians by distance

\begin{tabular}{|l|c|c|c|}
\hline & $\begin{array}{c}\text { Leading } \\
\text { participants }\end{array}$ & $\begin{array}{c}\text { Approaching } \\
\text { participants }\end{array}$ & Static obstacles \\
\hline The number of data & 24 & 3 & 60 \\
\hline Average distance $(\mathrm{m})$ & 1.90 & 3.97 & 4.58 \\
\hline Standard Deviation & 0.71 & 0.54 & 1.89 \\
\hline
\end{tabular}

Figure 10 plots the relative position of the fixated object in each observed fixation on another participant or a static obstacle. Positions of objects are relative to the position of the eye-tracker participant, and therefore the position of the participant is always (0.0). The walking direction of the participant is set to be toward $(1,0)$. In the calculation, we assumed that the participant always walked parallel to the longitudinal edge of the platform as the platform shape is a long rectangle. In the figure, filled-diamonds represent positions of static obstacles, with filled-squares representing leading participants and filled-triangles oncoming participants. The figure also includes "ignored participants" as well as "ignored obstacles" plotted with cross marks. These are other participants or static obstacles in the sight of the participant but not fixated because the participant fixated on another participant or static obstacle. Note that in some cases in which there was more than one participant or obstacle in the eye-tracker participant's sight, the eye-tracker participant at first fixated on one object (say, an obstacle) for a while and then fixated on another participant or obstacle although the obstacle was still in the eye-tracker participant's sight. In these cases, the first 
fixated obstacle was plotted in the figure as fixated but later plotted as an "ignored obstacle", which means that a line of consecutive diamond marks in the figure becomes a line of consecutive cross marks at a certain point. Figure 11 is a schematic representation of this.

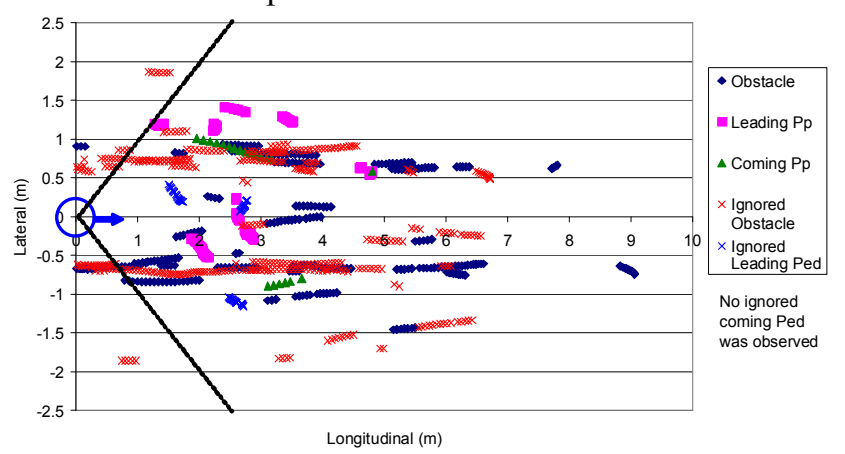

Fig. 10 Relative position of fixated or ignored objects

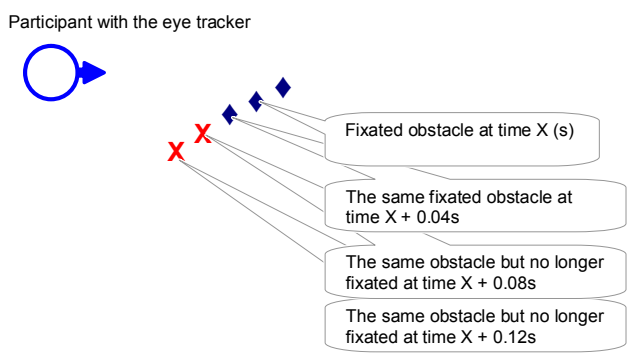

Fig. 11 Schematic representation of transition of an object from "fixated" to "ignored"

Interestingly, the figure shows that there were few ignored oncoming participants and obstacles in the area directly in front of the participant (longitudinal coordinate: 0.0 to around $4.5 \mathrm{~m}$, lateral coordinate: $0.5 \mathrm{~m}$ to $0.5 \mathrm{~m})$. Also we can observe that there was no fixated participant or obstacle in the area with an angle of $45^{\circ}$ or more from the walking direction of the participant.

\section{Discussion}

In this paper, we have explored the size and the shape of Information Process Space, in which a pedestrian takes account of other pedestrians and obstacles. We conducted an experiment wherein a participant wore an eye tracker and avoided collision with other participants and obstacles on the platform. The results led to 3 interesting insights on Information Process Space.

Firstly, we expected that the eye-tracker participant would frequently fixate on static objects or pedestrians. The results show, however, that there were also many fixations on the platform surface or the facing parapet.

Interestingly, when the participant first came to the platform before starting to walk, it was observed that the participant fixated on objects on the platform. It is speculated that the pedestrian with the eye tracker obtained the basic information about objects on the platform (e.g. the existence and the approximate location of other pedestrians and obstacles) before the start of 
each session, and during the experiment the pedestrian fixated on other pedestrians/obstacles when it was really necessary for collision avoidance. On the other hand, the pedestrian often fixated on the platform surface because walking safely on the platform surface may be an immediate issue for the pedestrian.

As mentioned in the Introduction section, Hoogendoorn et al suggested three levels of pedestrians' decision making process while walking: strategic, tactical and operational level $[5,13,16]$. A comparison between our results indicates that there may be one level below these three levels i.e. checking environments (e.g. surface) for safe walking. Indeed, this finding makes us recall an important fact: pedestrians take account not only of collision avoidance but also of immediate environmental hazards (e.g. a gap in the surface). It would be interesting to investigate how these levels interact with each other. Previous studies [17, 18] suggested that, in low lighting conditions, pedestrians fixate on "hazard" objects longer than "orientation" objects. It is speculated that the more attention a pedestrian pays to a hazard object, the less attention may be paid to other pedestrians or obstacles. There is room for further research into how to model pedestrians' attention in a low visibility environment.

Secondly, the results of our experiment give insights into the shape of Information Process Space. Many pedestrian simulations have assumed it is a semicircle, whereas the results imply that pedestrians are more interested in objects directly in front to which the relative lateral distance is small. Also, the participants seldom fixated on objects to which the angle from the walking direction was more than 45 degrees. These observations suggest that IPS may have a different form from a homogeneous semicircle. It seems that some areas, especially the exact front area, have higher priority in pedestrians' attention than other areas.

It is noteworthy that there was no fixation observed within the immediate vicinity (the area in front of the body within a radius of $1.5 \mathrm{~m}$ ). Patra and Vickers conducted a series of experiments in which participants were asked to approach and to step over an obstacle, or to step on specific locations [19, 20]. They found that participants fixated on the landing target on average two steps ahead. Two steps may be necessary for the human body to control limb trajectory and then to successfully step over/on a target. On the other hand, collision avoidance requires more movements and more time because a person needs to displace himself/herself away from an oncoming pedestrian or an obstacle. If there is an object directly in front to which the distance is less than $1.5 \mathrm{~m}$, it may be too late to fixate on another pedestrian or an obstacle and then avoid collision.

Thirdly, according to our results, durations of first fixations on leading participants were less than those on other oncoming participants. This could be because of a smaller possibility of colliding with a leading participant than with another oncoming participant. On the other hand, first fixations on static obstacles were slightly greater than those on oncoming participants. This could be because the participants also recognised static obstacles as objects that they might be more likely to collide with. We had expected that first fixations on oncoming participants might take place at an earlier stage than static obstacles because relative velocities to an oncoming participant were greater than those to static obstacles. However, our results did not match with this hypothesis. 
It should be noted that the fixations we observed might mainly be fixations at the collision avoidance or surface-check level. The shape of the information process space at the strategic or tactical level (to find a destination or to understand the approximate location of other pedestrians or obstacles) may be different. Also, this paper presents preliminary observations based on a limited number of samples. Future works order to understand the exact size of Information Process Space, further investigation is In order to understand the exact size and shape of Information Process Space, a further investigation is necessary.

\section{Conclusions}

This research empirically investigated the Information Process Space of pedestrians. Many pedestrian simulations assume a certain area, but little research has empirically investigated this. By analysing fixation behaviour of pedestrians using an eye camera, we examined the Information Process Space of pedestrians in the real world.

As pedestrian simulations become more and more sophisticated and used for various situations, it is necessary to understand how real pedestrians perceive other pedestrians and environments. Such knowledge would help further precise representation of pedestrians in simulations. Especially, as our study pointed out, pedestrians perceive not only other pedestrians or obstacles but also other environmental information, such as hazards on the floor surface. It would be of interest to model such whole environmental recognition systems and to integrate them into pedestrian simulations. In other words, pedestrian simulations can be a platform of knowledge of such human behaviour or human perception about environments.

\section{References}

1. Bierlaire, M., G. Antonini, and M. Weber. Behavioral dynamics for pedestrians. in 10th International Conference on Travel Behavior Research. 2003. Lucerne.

2. A.Thompson, P., Developing New Techniques for Modelling Crowd Movement. 1994, University of Edinburgh. p. 220.

3. Antonini, G., M. Bierlaire, and M. Weber. Simulation of Pedestrian Behaviour using a Discrete Choice Model Calibrated on Actual Motion Data. in Swiss Transport research Conference - Mobility Session 2004 conference. 2004.

4. FEURTEY, F., Simulating the Collision Avoidance Behavior of Pedestrians, in School of Engineering Department of Electronic Engineering. 2000, The University of Tokyo: Tokyo.

5. Hoogendoorn, S. and P.H.L. Bovy, Simulation of pedestrian flows by optimal control and differential games. OPTIMAL CONTROL APPLICATIONS AND METHODS, 2003. 24: p. 153-172.

6. Yamori, K., Going With the Flow: Micro-Macro Dynamics inthe MAcrobehavioral Patterns of Pedestrian Crowds. Psychological Review, 1998. 105(3): p. 530-557.

7. Blue, V.J. and J.L. Adler, Cellular automata microsimulation for modeling bi-directional pedestrian walkways. Transportation Research Part B: Methodological, 2001. 35(3): p. 293-312. 
8. Dijkstra, J., A.J. Jessurun, and H.J.P. Timmermans. A Multi-Agent Cellular Automata System for Visualising Simulated Pedestrian Activity. in Theoretical and Practical Issues on Cellular Automata, Proceedings of the Fourth International Conference on Cellular Automata for Research and Industry. 2000. Karlsruhe, Germany: Springer Verlag.

9. Lakoba, T.L., D.J. Kaup, and N.M. Finkelstein, Exploration of the parameter range of a continuous-space, agent-based model for pedestrian evolution. 2003.

10. Lakoba, T.I., D.J. Kaup, and N.M. Finkelstein, Modifications of the Helbing-Molna'r-Farkas-Vicsek Social Force Model for Pedestrian Evolution. SIMULATION, 2005. 81(5): p. 339-352.

11. Blue, V.J. and J.L. Adler, Flow capacities from cellular automata modelling of proportional splits of pedestrians by diretions, in Pedestrian and Evacuation Dynamics, M. Schreckenberg and S.D. Sharma, Editors. 2002, Springer: Berlin. p. 115 -- 122.

12. Turner, A. and A. Penn, Encoding natural movement as an agentbased system: an investigation into human pedestrian behaviour in the built environment. Environment and Planning B: Planning and Design, 2002. 29(4): p. 473-490.

13. HOOGENDOORN, S.P. and P.H.L. BOVY, Pedestrian Travel Behavior Modeling. Networks and Spatial Economics, 2005. 5: p. 193-216.

14. Helbing, D., I. Farkas, and T. Vicsek, Simulating dynamical features of escape panic, in Nature. 2000. p. 487-490.

15. Suzuki T, Okazaki S, 2002, Visual search while climbing or descending the staircases at a subway station, Journal of architecture, planning and environmental engineering, 558, pp151-158, in Japanese

16. Hoogendoorn S, Bovy P, Daamen W, 2002, "Microscopic pedestrian wayfinding and dynamics modeling", in M. Schreckenberg and S. Sharma (Eds.) Pedestrian and Evacuation, Springer, pp123-154

17. Fujiyama T, 2006, Investigating density effects on the "awareness" area of pedestrians using an eye tracker CTS Working Paper 2006/4, Centre for Transport Studies, University College London, London, ISSN 1747-6232

18. Fujiyama T, Childs CR, Boampong D, Tyler N, 2007, How do elderly pedestrians perceive hazards in the street? -An initial investigation towards development of a pedestrian simulation that incorporates reaction of various pedestrians to environments- Paper presented to 11th International Conference on Mobility and Transport for Elderly and Disabled People, Montreal, Canada, June 2007

19. Patra AE, Vickers JN, 1997, Where and when do we look as we approach and step over an obstacle in the travel path?, Neuroreport, 8, pp3661-3665

20. Patra AE, Vickers JN, 2003, How far ahead do we look when required to step on specific locations in the travel path during locomotion?, Experimental Brain Research, 148, pp133-138 